\title{
Detection of underlying dementia in bullous pemphigoid patients using cognitive evaluation tests: a multicenter case-control study
}

\author{
Wenling Zhao ${ }^{1,2 \#}$, Yiman Wang ${ }^{1 \#}$, Xuming Mao ${ }^{3}$, Aimee S. Payne ${ }^{3}$, Suying Feng ${ }^{4},{\text { Wei } \text { Li }^{5} \text {, Yanhong Wang }}^{6}$, \\ Di Wang ${ }^{1}$, Hongzhong Jin ${ }^{1}$, Jing Yuan ${ }^{7}, \mathrm{Li} \mathrm{Li}^{1}$ \\ ${ }^{1}$ Department of Dermatology, Peking Union Medical College Hospital, Chinese Academy of Medical Sciences and Peking Union Medical College, \\ Beijing, China; ${ }^{2}$ Department of Dermatology, Shunyi Maternal and Children's Hospital of Beijing Children's Hospital, Beijing, China; ${ }^{3}$ Department \\ of Dermatology, University of Pennsylvania, Philadelphia, PA, USA; ${ }^{4}$ Institute of Dermatology, Department of Dermatology, Chinese Academy of \\ Medical Sciences and Peking Union Medical College, Nanjing, China; ${ }^{5}$ Department of Dermatology, West China Hospital, Sichuan University, \\ Chengdu, China; ${ }^{6}$ Department of Epidemiology and Bio-statistics, Institute of Basic Medical Sciences, China Academy of Medical Sciences and \\ Peking Union Medical College, Beijing, China; ${ }^{7}$ Department of Neurology, Chinese Academy of Medical Sciences and Peking Union Medical \\ College Hospital, Chinese Academy of Medical Sciences and Peking Union Medical College, Beijing, China \\ Contributions: (I) Conception and design: L Li, J Yuan, W Zhao; (II) Administrative support: W Zhao, Y Wang; (III) Provision of study materials or \\ patients: L Li, S Feng, W Li, H Jin; (IV) Collection and assembly of data: W Zhao, D Wang; (V) Data analysis and interpretation: J Yuan, X Mao, AS \\ Payne, Y Wang, W Zhao; (VI) Manuscript writing: All authors; (VII) Final approval of manuscript: All authors. \\ \#These authors contributed equally to this work. \\ Correspondence to: Li Li, MD; Jing Yuan, MD. Peking Union Medical College Hospital, Chinese Academy of Medical Sciences and Peking Union \\ Medical College, Beijing, China. Email: lilipumch2007@sina.com; yuanjing@pumch.cn.
}

Background: Preliminary observation in clinical practice showed that subjective neurocognitive complaints are relatively common in bullous pemphigoid (BP) patients. Yet, little has been done to investigate the neurocognitive status in BP.

Methods: This is a multicenter observational case-control study comprised of $61 \mathrm{BP}$ patients and 65 matched control subjects from 3 medical centers in China from 2014 to 2019. To evaluate the cognitive function between BP patients and matched controls, all the subjects finished the mini-mental state examination (MMSE) and montreal cognitive assessment (MoCA).

Results: The overall scores were significantly lower in BP than in controls $\left(\mathrm{P}_{\mathrm{MoCA}}<0.001\right)$. The percentage of patients screened positive for cognitive impairment in the BP group was significantly higher than in the control group $\left(\mathrm{P}_{\mathrm{MMSE}}<0.001, \mathrm{P}_{\mathrm{MoCA}}<0.001\right)$. On multivariate analysis, $\mathrm{BP}$ was associated with increased odds of cognitive impairment by MMSE and MoCA $\left(\mathrm{P}_{\mathrm{MMSE}}=0.017, \mathrm{P}_{\mathrm{MoCA}}=0.007\right)$.

Conclusions: BP patients had decreased cognitive abilities and were at a higher risk of cognitive impairment. The evaluation of cognitive impairment may be warranted for BP patients in clinical practice for early diagnosis and therapy of dementia.

Keywords: Alzheimer's disease (AD); bullous pemphigoid (BP); cognitive impairment; mini-mental state examination (MMSE); montreal cognitive assessment (MoCA)

Submitted Feb 04, 2020. Accepted for publication Sep 06, 2020.

doi: 10.21037/atm-20-1319

View this article at: http://dx.doi.org/10.21037/atm-20-1319

\section{Introduction}

Bullous pemphigoid (BP) is an autoimmune skin disease more commonly found in the elderly (1). We and others have revealed that patients with $\mathrm{BP}$ are more likely to develop neurological diseases (ND), including cerebrovascular diseases, Parkinson's disease, and multiple sclerosis (2). ND can progress silently without being noticed by patients, their relatives, or even medical professionals for 
many years. Interestingly, dermatologists are often the first to realize the neurological abnormality in BP patients (1-4).

It has been previously reported that several ND with inflammation or degeneration in the central nervous system were related to BP (5). Recently, we have shown that a significantly high level of BP antibodies could be detected in patients with stroke (6). Although the mechanism linking $\mathrm{BP}$ to ND is largely unclear, the current hypothesis is that damage of the brain tissue leads to exposure of $\mathrm{BP}$ antigen 1 (BPAG1 or BP230) and BP antigen 2 (BPAG2 or BP180) to the immune system and production of antiBP180 antibodies that may cause blister formation at the basement membrane $(7,8)$. Several lines of evidence have also suggested that the antigens in the central nervous system start to be exposed to the immune system because of the gradual dysfunction of the blood-brain barrier during aging $(9,10)$. In brains of aged mice, BP180 appeared highly expressed in the hippocampal area, which is one of the most common lesional locations of Alzheimer's disease (AD) (9), a hallmark ND with dementia in the elderly.

Neurocognitive impairment, including mild cognitive impairment (MCI) and dementia, is commonly found in a variety of ND $(11,12)$. MCI is widely viewed as the intermediate phase, from normal cognition to dementia. MCI may further progress to dementia (13), with a progression rate of approximately $5-15 \%$ per year (14). Although up to $38 \%$ of MCI patients might revert to normal cognition, as high as $65 \%$ of them eventually developed MCI or dementia (15), suggesting the importance of early evaluation and management of MCI.

To date, only a few studies have explored the potential correlation between BP and dementia $(16,17)$, and BP complicated with dementia could imply a much higher risk of relapse, which potentially leads to an even worse prognosis for BP (18). To quantitatively determine the neurocognitive function in $\mathrm{BP}$, we screened the patients using two cognitive function instruments including minimental state examination (MMSE) and montreal cognitive assessment (MoCA).

We present the following article in accordance with the STROBE reporting checklist (available at http://dx.doi. org/10.21037/atm-20-1319).

\section{Methods}

\section{Patients}

Our study is a multicenter case-controlled study with all subjects that were recruited from 3 hospitals in China between 2014 and 2019 (Peking Union Medical Hospital, Institute of Dermatology, West China Hospital). After screening with the following inclusion and exclusion criteria, a total of 61 patients with BP and 65 controls matched in age, gender, and education were included in the study. The diagnosis of $\mathrm{BP}$ was made according to the British Association of Dermatologists' guidelines for the management of BP (19) and confirmed by an experienced dermatologist according to the following inclusion criteria: (I) typical clinical manifestation (tense papules or blisters on erythema); (II) pathology features (subepidermal blister); (III) positive direct/indirect immunofluorescence (DIF/IIF) and anti-BP180/BP230 antibodies in freshly collected serum by Enzyme-Linked Immunosorbent Assay (ELISA). The BP180 and BP230 ELISA kit (MESACUP BP180 TEST, CODE No. 7695E; MESACUPBP230, CODE No. 7613E) were purchased from Medical \& Biological Laboratories CO. LTD. Exclusion criteria for both groups if the patients with (I) an ambiguous diagnosis of BP; (II) mental disorders such as schizophrenia; (III) disorders of consciousness like delirium; (IV) inability to cooperate because of deafness, blindness or other somatic diseases; (V) a Hamilton Depression Scale (HAMD-17) score greater than 17 to exclude depression-related cognitive dysfunction (20); (VI) other cognitive disorders, such as syphilis infection, vitamin B12 deficiency, and thyroid dysfunction; (VII) refusal to participate or continue the evaluation; (VIII) a history of medication affecting cognition.

This study was conducted in accordance with the Declaration of Helsinki (as revised in 2013). The study was approved by the institutional research ethics committee of Peking Union Medical College Hospital (S-K265), and informed consent was obtained from all the patients.

\section{Cognition assessment}

The MMSE and MoCA are screening tests for cognitive impairment recommended by the Dementia and Cognitive Society of Neurology Committee in the Chinese Medical Association and Alzheimer's Disease. Both tests and neurological physical examinations (with medical record reviews) were performed by dermatologists trained by neurologists. Subjects screened positive on either assessment were considered to have neurocognitive impairment and referred to neurologists for further evaluations. Both the raters and the neurologists were blinded to the patients' groups. 


\section{MMSE}

MMSE is the most widely used screening test for cognitive impairment, which includes evaluations in 7 domains: orientation, memory, attention, calculation, language, executive function, and visual spatial ability with a total score of 30. A higher score indicates better cognitive function. The revised Chinese version of the MMSE was used for this study. Cut-offs for screening positive are set according to educational level: $\leq 17$ points for illiterate, 20 points for primary education, and 24 points for high school or higher education (21). If the result is below the threshold, it means the patient has cognitive impairment.

\section{MoCA}

The MoCA scale covers evaluations in 8 domains: attention, executive function, memory, language skills, spatial structure, abstract thinking, calculation, and orientation. The cut-off for screening impairment in the revised Chinese version of MoCA is equal or lower than 26, with an additional 1 point for those whose educational level is equal to or lower than 12 years (22). If the result is below the threshold, it means the patient has cognitive impairment.

\section{Statistical analysis}

Data management and analyses were performed using the IBM SPSS statistics 20.0 software. The enumeration data were described with frequency (proportion). The Chisquare test was used to compare the difference between the two groups. The quantitative data were described using mean (standard deviation) or median (quantiles) for normal or abnormal distribution, respectively. A non-parametric test (Mann-Whitney $U$ ) was used to compare the difference between the two groups for normal or abnormal distributed quantitative data. The associations between $\mathrm{BP}$ as a dependent variable (yes or no) and independent variables including gender (male or female), age ( $\geq 70$ years or $<70$ years), whether combined with ND and screening test results (positive or negative, dichotomous variables), were evaluated using logistic regression. In the multivariate analysis logistic regression model, the associations between $\mathrm{BP}$ as a dependent variable (yes or no) and independent variables including screening test results (positive or negative), adjusted for gender (male or female), age ( $\geq 70$ years or $<70$ years) and ND history were determined. The associations between screening test scores as the dependent variable (scores, continuous variable) and anti-BP180 antibody levels, anti-BP230 antibody levels and IIF titers as the independent variables (examined before treatment, continuous variable) were evaluated by Spearman's Correlation, stratified by age (continuous variable), gender (male or female), and ND history. The statistical significance level was set to $\mathrm{P}<0.05$.

\section{Results}

\section{Basic characteristics and neurological assessment in BP and control groups}

A total of 61 patients with BP completed the cognitive screening test and entered the final analysis (Figure 1), together with 65 matched controls. There was no significant difference between the two groups in gender, age, or years of education ( $\mathrm{P}=0.95,0.074$, and 0.741 , respectively) (Table 1). The BP group demonstrated a significantly higher percentage of ND $(14 / 61,23 \%)$ than the control group $(4 / 65,6 \%, \mathrm{P}<0.001)$. The percentage of $\mathrm{BP}$ patients who were screened positive by MMSE and MoCA was higher than that of the control group (MMSE: 17 vs. 3, 28\% vs. 5\%, $\mathrm{P}<0.001$; MoCA: 54 vs. $42,89 \%$ vs. $65 \%, \mathrm{P}<0.001)$. The overall MoCA score of BP patients (adjusted by education years) was significantly lower than that of the control group (19 vs. 24, $\mathrm{P}<0.001$ ). In cognitive domain evaluations, the BP group and control group had remarkable differences in all domains except memory. In the memory domain, a significant difference was found by the MoCA $(\mathrm{P}<0.001)$ but not the MMSE scale $(\mathrm{P}=0.138)$. The score of $\mathrm{BP}$ patients in the HAMD-17 test was significantly higher than the control group \{median [(25\% interquartile range (IQR)], 5 (IQR 2) vs. 3 (IQR 1), $\mathrm{P}=0.039$ \} even within subjects whose HAMD17 score was below 17 .

\section{The association between BP and MMSE/MoCA screening results}

On multivariate analysis, after adjustment for age, gender and presence of ND, BP patients had a significantly higher risk of cognitive impairment than controls, by either MMSE [odds ratio (OR) 6.38, 95\% CI: 1.39-29.32 or MoCA (OR 3.78, , 95\% CI: 1.44-9.96] (Table 2). BP was associated with positive screening results $\left(\mathrm{P}_{\mathrm{MMSE}}=0.017\right.$, $\left.\mathrm{P}_{\mathrm{MoCA}}=0.007\right)$. When compared BP patients with ND and control patients with ND, even when both groups had matched in ND, BP patients had significantly lower 


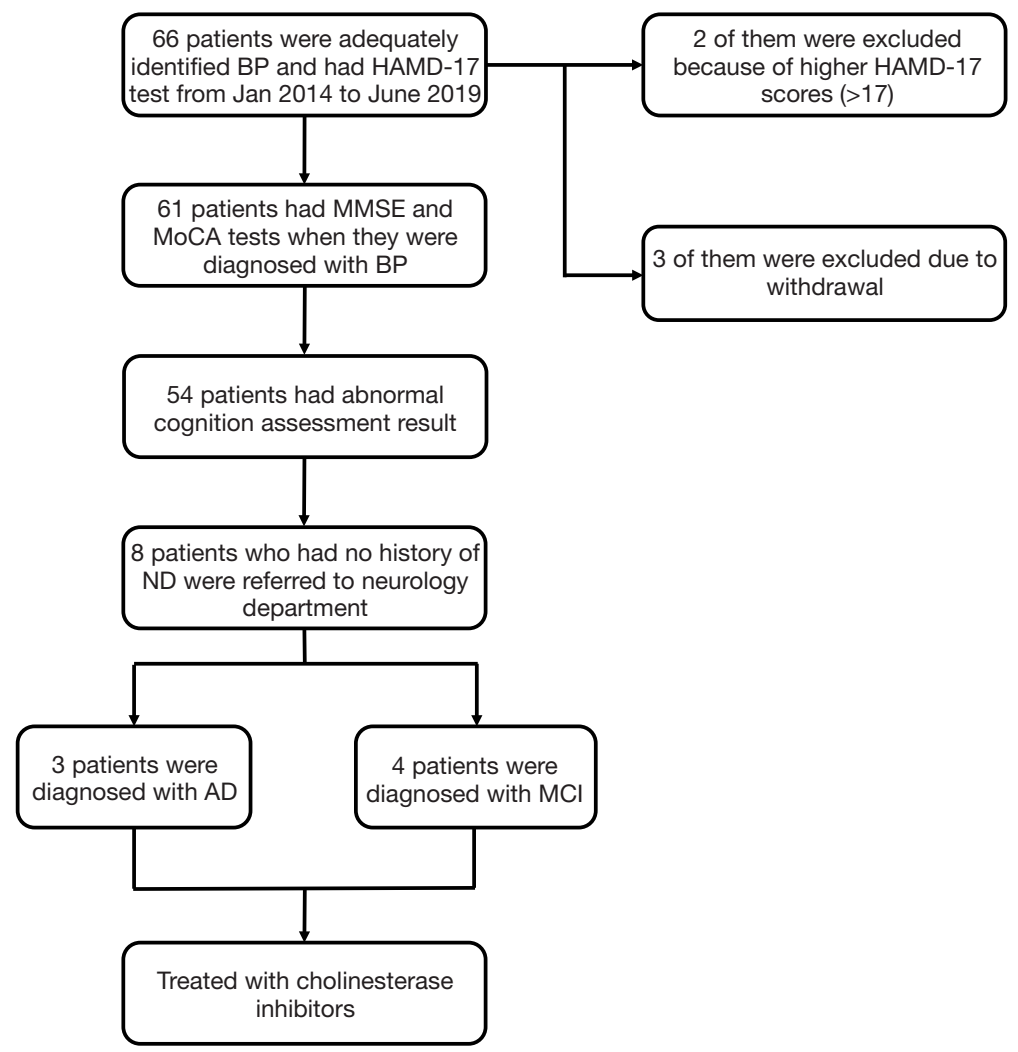

Figure 1 Study flow diagram of BP patients.

cognitive scores in both MMSE and MoCA $\left(\mathrm{P}_{\text {MMSE }}=0.047\right.$, $\mathrm{P}_{\text {MoCA }}=0.055$ ) (Figure 2). As for subjects without $\mathrm{ND}, \mathrm{BP}$ without ND had a significantly lower score than Control without ND on MoCA test $\left(\mathrm{P}_{\mathrm{MoCA}}<0.001\right)$ (Figure $\left.2 B\right)$. This suggests $\mathrm{BP}$ might be an independent risk factor for cognitive impairment, particularly MCI, regardless of ND.

\section{The association between MMSE/MoCA scores and anti- BP180 antibody level/anti-BP230 antibody level/IIF titer}

The associations between the MMSE/MoCA scores and anti BP180/230 antibody levels or IIF titers (before treatment) were evaluated in BP patients. We found that the MMSE score and MoCA score are negatively related with IIF titer $\left(\mathrm{r}_{\mathrm{MMSE}}=-0.452, \mathrm{P}_{\mathrm{MMSE}}=0.004 ; \mathrm{r}_{\mathrm{MoCA}}=-0.488, \mathrm{P}_{\mathrm{MoCA}}=0.002\right)$. There was no significant correlation between these screening test scores and anti-BP180/230 antibody levels. Further analysis based on age, gender and neurological history (Table 3) revealed that MMSE and MoCA scores were negatively correlated with IIF titers when patients were younger $(<70$ years $)\left(\mathrm{P}_{\mathrm{MMSE}}=0.001, \mathrm{P}_{\mathrm{MoCA}}=0.019\right)$, female $\left(\mathrm{P}_{\text {MMES }}=0.004, \mathrm{P}_{\mathrm{MoCA}}=0.001\right)$ or without ND $\left(\mathrm{P}_{\text {MMSE }}=0.004\right.$,
$\left.\mathrm{P}_{\mathrm{MoCA}}=0.007\right)$. The MMSE score was negatively related to IIF titers in male patients $(\mathrm{P}=0.024)$. The MoCA score was negatively related to IIF titer in patients with ND history $(\mathrm{P}=0.036)$. The MMSE and MoCA scores were negatively related to anti-BP180 IgG antibody in patients without ND history $\left(\mathrm{P}_{\text {MMSE }}=0.026, \mathrm{P}_{\mathrm{MoCA}}=0.012\right)$.

A total of 38 subjects with $\mathrm{BP}$ and screened positive for cognitive impairment were initially recommended to refer to the Department of Neurology for further examinations, including detailed neuropsychological battery, ApoE genotype, electroencephalogram and head CT/MRI scan. 12 patients were excluded due to a prior history of ND diagnosis by neurologists. Of the 8 patients who have no history of $\mathrm{ND}$ and were eventually referred to the neurologists by us, 4 patients were diagnosed with MCI, 3 patients with $\mathrm{AD}$, and one with normal. Therapies including cholinesterase inhibitors were prescribed for $\mathrm{AD}$ patients.

\section{Discussion}

To our knowledge, this is a novel multicenter retrospective case-control study aimed to quantitatively determine 
Table 1 The comparison between BP group and control group

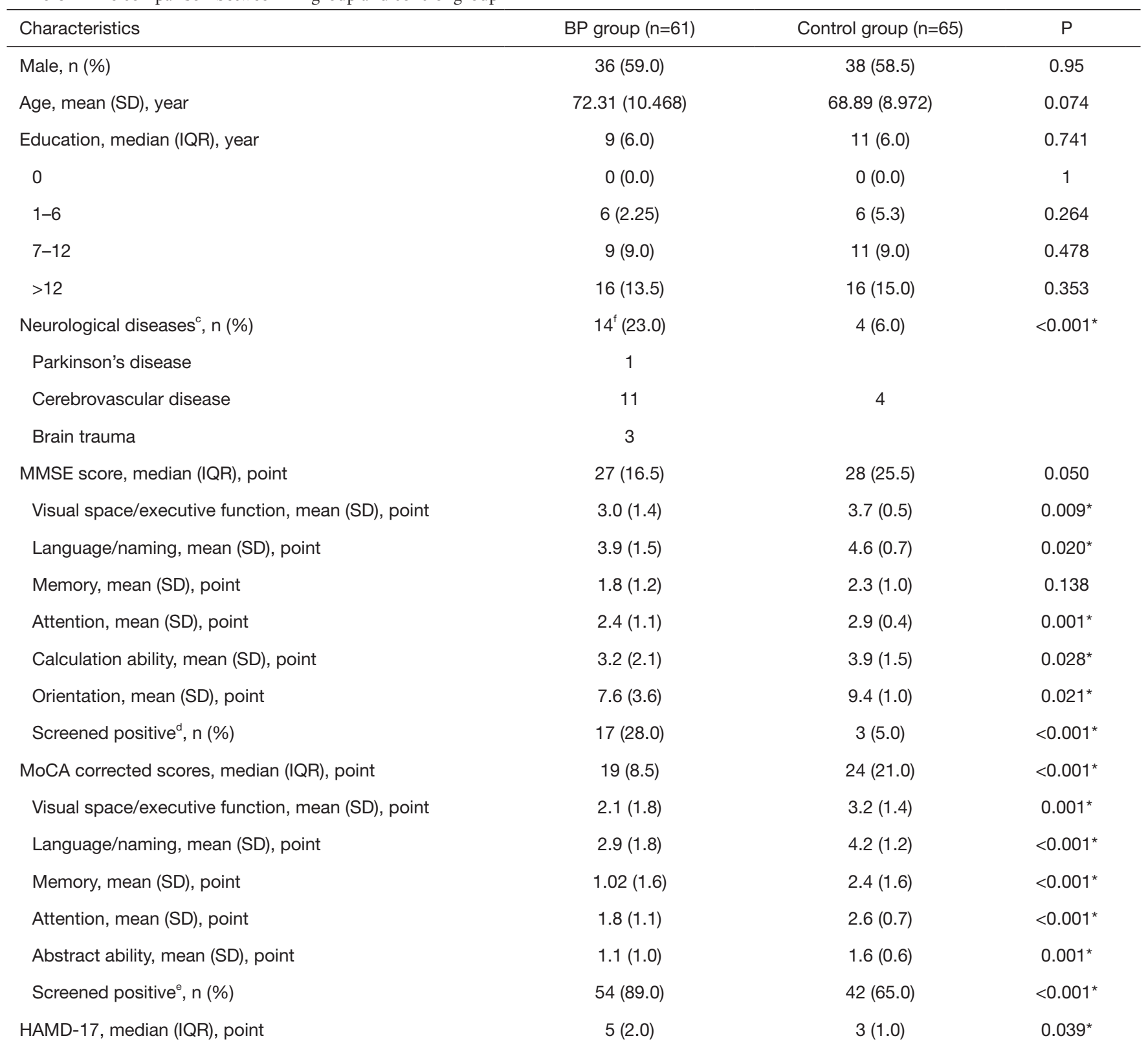

${ }^{*}, \mathrm{P}<0.05 .{ }^{\circ}$, the combined neurological diseases include cerebrovascular disease, brain trauma, Parkinson's disease, and amyotrophic lateral sclerosis; ${ }^{d}$, cognitive impairment was determined according to MMSE score and education level, which was: the illiteracy group $\leq 17$, the primary group $\leq 20$, the secondary school or the above group was $\leq 24$; ${ }^{\text {, the }}$, MoCA score was adjusted when the subjects had education for less than 12 years: one point was added to the total score while no more than 30 points should be guaranteed. Impairment of cognitive function was assessed according to the score of correction, and the demarcation value of the damage was $\leq 26$; ${ }^{f}$, one patient has a history of cerebrovascular disease and brain trauma. BP, bullous pemphigoid; MMSE, mini-mental state examination; MoCA, Montreal cognitive assessment; HAMD-17, Hamilton Depression Scale. SD, standard deviation; IQR, interquartile range. 
Table 2 Multivariate logistic regression analysis of BP group and control group

\begin{tabular}{|c|c|c|c|c|}
\hline Characteristics & \multicolumn{2}{|c|}{ Model 1 (age, gender, history of ND, MMSE) } & \multicolumn{2}{|c|}{ Model 2 (age, gender, history of ND, MoCA) } \\
\hline \multicolumn{5}{|l|}{ Age, years } \\
\hline$<70$ & Reference & & Reference & \\
\hline$\geq 70$ & $0.89(0.41-1.94)$ & 0.769 & $0.93(0.43-2.04)$ & 0.855 \\
\hline Female & Reference & & Reference & \\
\hline Male & $1.16(0.54-2.46)$ & 0.708 & $1.22(0.57-2.60)$ & 0.615 \\
\hline \multicolumn{5}{|l|}{ History of ND } \\
\hline No & Reference & & Reference & \\
\hline No & Reference & & Reference & \\
\hline Yes & 6.38 (1.39-29.32) & $0.017^{*}$ & $3.78(1.44-9.96)$ & $0.007^{*}$ \\
\hline
\end{tabular}

*, $\mathrm{P}<0.05$. OR, odds ratio; $\mathrm{Cl}$, confidence interval; BP, bullous pemphigoid; ND, neurological diseases; MMSE, mini-mental state examination; MoCA, Montreal cognitive assessment.
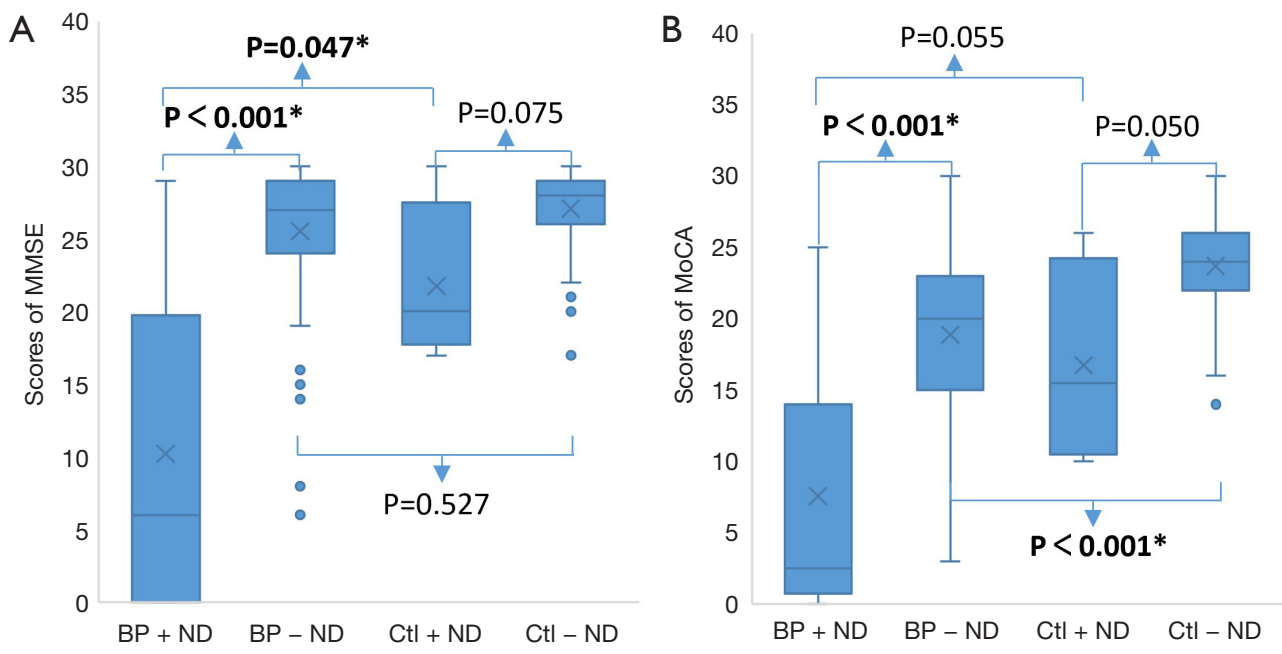

Figure 2 The effects of BP and ND on patients' cognitive function. The patients with (+ND) or without neurological diseases (-ND) in the BP group (BP) or control group (Ctl) were further compared in neurocognitive functions (scores) by MMSE (A) or MoCA (B), respectively. *, statistical significance. $\mathrm{BP}+\mathrm{ND}=$ the $\mathrm{BP}$ patients with $\mathrm{ND} ; \mathrm{BP}-\mathrm{ND}=$ the $\mathrm{BP}$ patients without $\mathrm{ND} ; \mathrm{Ctl}+\mathrm{ND}=$ the patients from control group with ND; $\mathrm{Ctl}-\mathrm{ND}=$ the patients from control group without ND.

cognitive impairment and dementia in BP patients. In accordance with previous findings (10), we found that the percentage of BP patients with ND as a combination was significantly higher than that in controls. To better estimate the risk of cognitive impairment in BP, we applied both MMSE and MoCA to define the cognitive status in all control and BP patients. Remarkably, we found neurocognitive impairment could be identified in up to $28 \%$ for MMSE and $89 \%$ for MoCA of BP patients (Table 1).

Notably, marked differences could be observed in sub- 
Table 3 Correlation analysis of the anti-BP antibody value by ELISA or titer by IIF with the MMSE score and MoCA score in BP patients

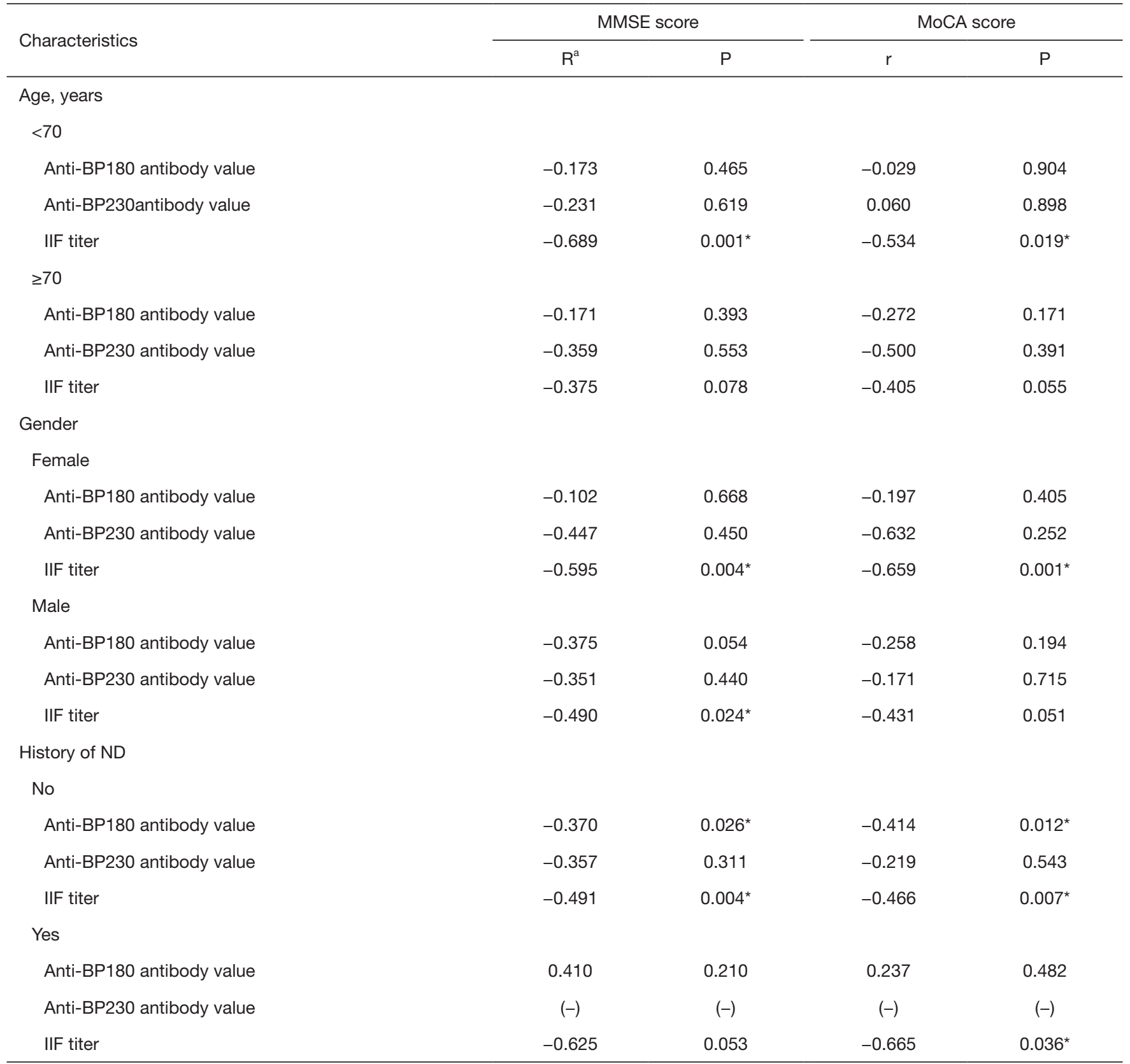

${ }^{a}$, correlation coefficient. * $\mathrm{P}<0.05$. MMSE, mini-mental state examination; MoCA, Montreal cognitive assessment; ND, neurological diseases; IIF, indirect immunofluorescence.

domains between MoCA and MMSE when we compared the BP and control groups (Table 1). In "memory" sections, the MoCA scale study showed a significant difference between the groups while the MMSE scale did not. This might be due to the difference in scale design between MMSE and MoCA. Studies have shown that the MMSE scale can effectively screen dementia patients, but has a limited role in differentiating healthy subjects from mild cognitive impairment patients $(23,24)$. MoCA scale tends to have higher sensitivity for the diagnosis of MCI (25-28), which explained the difference between the MMSE scores and MoCA scores (Table 1). 
Besides, despite of the difference in screening sensitivity between the two instruments, we found that patients with $\mathrm{BP}$ appeared to have significantly higher risk of positive screening results for cognitive impairment, either MCI or dementia (Table 2), suggesting BP may impair the cognitive function in patients by targeting various areas in the brain by the $\mathrm{BP}$ autoantibodies.

Next, we compared the neurocognitive scores from either MMSE or MoCA. We found the overall score in the BP group was significantly lower than the control group. Because a high percentage of BP patients have ND that could significantly decrease the cognitive function (29), we also compared the scores of patients with or without ND. In control groups without $\mathrm{BP}$, there was no statistically significant difference in cognitive scores between groups with and without ND (Figure 2). Intriguingly, we found that BP patients with ND had significantly lower scores than those with BP alone, indicating that ND could further impair the cognitive function. To further test the possibility that reduction of cognitive function in BP might be excluded due to ND, the scores of BP without ND group and Control without ND group were compared, a significantly lower cognitive score by MoCA in the $\mathrm{BP}$ group suggests BP may cause neurocognitive dysfunction without preexisting ND. There was also a slight but noticeable decrease in the cognitive score by MMSE, albeit with no statistical significance, which might be explained by the relatively lower screening sensitivity of MMSE when compared to $\mathrm{MoCA}(30)$. Altogether, these data suggest that BP might be related to cognitive impairment independent of ND. This notion was further supported by the fact that a markedly high ratio of patients (7/8), who were screened with positive cognitive impairment and referred to the neurologists after being excluded from any existing ND, were verified to have neurocognitive dysfunctions. These included 3 patients with $\mathrm{AD}$ and 4 patients with MCI, who were immediately managed and closely followed by the neurologists.

Although our statistical analysis showed no significant correlation between anti-BP180/230 ELISA value and the cognitive function, which was consistent with previous studies showing BP180/230 ELISA value not related to $\mathrm{BP}$ with or without ND (31), the IIF titer was found to be negatively correlated with MMSE/MoCA score. It is probably because the commercially available ELISA test kit can only detect autoantibodies that bind to the epitopes located in the BP180 NC16A region, while sera from a subset of ELISA-negative BP patients might contain the antibodies reacting only with epitopes outside the NC16A domain (32). While age and ND are known risk factors for cognitive impairment $(23,24)$, the role of gender is unknown. We found that the IIF titers in female patients were negatively correlated with the MMSE/MoCA scores. Besides, the median MMSE/MoCA scores of female patients were lower than male patients, although this difference was not statistically significant $\left(\mathrm{P}_{\mathrm{MMSE}}=0.488\right.$, $\left.\mathrm{P}_{\mathrm{MOCA}}=0.103\right)$.

Given that the onset of dementia in BP is relatively insidious, it is often overlooked in clinical practice. Dementia in BP may start with MCI that may have a high risk of developing into dementia (13). Diagnosis of dementia has stringent and complicated criteria such as that of the International Classification of Diseases tenth version or the Diagnostic and Statistical Manual fourth version. A comprehensive classification of dementia requires multiple evaluations, including cognitive function and brain imaging tests. The MMSE and MoCA tests are simple and feasible for use in clinical practice, by which we may decide whether we should refer BP patients to neurologists. Patients with early detection of cognitive impairment and even dementia have been reported to be more effectively treated and thus could improve the quality of life (33).

This is a multicenter trial study, albeit with relatively small sample size. Also, the retrospective nature of the current work could lead to a biased interpretation of the data. In the future study, a group of patients with a larger sample size with a prospective design might be necessary to validate how these screening tests that may result in early diagnosis. Our results indicate that early medical interventions based on early neurocognitive screening could improve the life quality and affect the life span of patients with BP.

\section{Conclusions}

Our study has demonstrated that BP patients have a high risk of cognitive dysfunction and that dermatologists consider applying the MMSE or MoCA scales for BP patients, who should be referred to neurologists if the screening tests are positive. Even for BP patients without $\mathrm{ND}$, they may have MCI or subclinical ND that warrant further evaluation. Also, within the groups of patients with ND, BP with ND had lower cognitive scores with the matched group with ND, which suggests that BP may be an independent risk factor for cognitive impairment with or without ND. Although future studies with a larger sample size might be necessary to confirm our results, our data 
from a multi-centric study suggest that a simple yet effective neuropsychological screening test such as MMSE and MoCA can be valuable in helping improve the overall life quality for $\mathrm{BP}$ patients who have a chronic disease course.

\section{Acknowledgments}

Funding: This work was supported by the National Natural Science Foundation of China (No. 81972945, to L Li); the Milstein Medical Asian American Partnership Foundation (2017 Dermatology to L Li); the National Key Research and Development Program of China Grant (No. 2016YFC0901500, L Li); the Education Reform Projects of Peking Union Medical College (No. 2016zlgc0106, to L $\mathrm{Li}$ ); the Funds of Shunyi District for Health Improvement and Research (No. Wsjkfzkyzx-2019-y-07, to W Zhao).

\section{Footnote}

Reporting Checklist: The authors have completed the STROBE reporting checklist. Available at http://dx.doi. org/10.21037/atm-20-1319

Data Sharing Statement: Available at http://dx.doi. org/10.21037/atm-20-1319

Conflicts of Interest: All authors have completed the ICMJE uniform disclosure form (available at http://dx.doi. org/10.21037/atm-20-1319). The authors have no conflicts of interest to declare.

Ethical Statement: The authors are accountable for all aspects of the work in ensuring that questions related to the accuracy or integrity of any part of the work are appropriately investigated and resolved. This study was conducted in accordance with the Declaration of Helsinki (as revised in 2013). The study was approved by the institutional research ethics committee of Peking Union Medical College Hospital (S-K265), and informed consent was obtained from all the patients.

Open Access Statement: This is an Open Access article distributed in accordance with the Creative Commons Attribution-NonCommercial-NoDerivs 4.0 International License (CC BY-NC-ND 4.0), which permits the noncommercial replication and distribution of the article with the strict proviso that no changes or edits are made and the original work is properly cited (including links to both the formal publication through the relevant DOI and the license). See: https://creativecommons.org/licenses/by-nc-nd/4.0/.

\section{References}

1. Pietkiewicz P, Gornowicz-Porowska J, BowszycDmochowska $M$, et al. Bullous pemphigoid and neurodegenerative diseases: a study in a setting of a Central European university dermatology department. Aging Clin Exp Res 2016;28:659-63.

2. Tarazona MJ, Mota AN, Gripp AC, et al. Bullous pemphigoid and neurological disease: statistics from a dermatology service. An Bras Dermatol 2015;90:280-2.

3. Gambichler T, Segert H, Höxtermann S, et al. Neurological disorders in patients with bullous pemphigoid: clinical and experimental investigations. J Eur Acad Dermatol Venereol 2015;29:1758-62.

4. Teixeira VB, Cabral R, Brites MM, et al. Bullous pemphigoid and comorbidities: a case-control study in Portuguese patients. An Bras Dermatol 2014;89:274-8.

5. Försti AK, Jokelainen J, Ansakorpi H, et al. Psychiatric and neurological disorders are associated with bullous pemphigoid - a nationwide Finnish Care Register study. Sci Rep 2016;6:37125.

6. Wang Y, Mao X, Wang D, et al. Anti-BP180 Autoantibodies Are Present in Stroke and Recognize Human Cutaneous BP180 and BP180-NC16A. Front Immunol 2019;10:236.

7. Chen JB, Li L, Chen J, et al. Sera of elderly bullous pemphigoid patients with associated neurological diseases recognize bullous pemphigoid antigens in the human brain. Gerontology 2011;57:211-6.

8. Li L, Chen J, Wang B, et al. Sera from patients with bullous pemphigoid (BP) associated with neurological diseases recognized $\mathrm{BP}$ antigen 1 in the skin and brain. $\mathrm{Br}$ J Dermatol 2009;160:1343-5.

9. Liu YD, Zhao WL, Zuo YG, et al. Research progress on association between bullous pemphigoid and neurological diseases. Chin J Mult Organ Dis Elderly 2016;15:633-5.

10. Brick KE, Weaver CH, Savica R, et al. A populationbased study of the association between bullous pemphigoid and neurologic disorders. J Am Acad Dermatol 2014;71:1191-7.

11. Burton L, Tyson SF. Screening for cognitive impairment after stroke: A systematic review of psychometric properties and clinical utility. J Rehabil Med 2015;47:193-203.

12. Moyer VA. U.S. Preventive Services Task Force. Screening for cognitive impairment in older adults: U.S. Preventive 
Services Task Force recommendation statement. Ann Intern Med 2014;160:791-7.

13. Langa KM, Levine DA. The diagnosis and management of mild cognitive impairment: a clinical review. JAMA 2014;312:2551-61.

14. Petersen RC, Roberts RO, Knopman DS, et al. Mild cognitive impairment: ten years later. Arch Neurol 2009;66:1447-55.

15. Roberts RO, Knopman DS, Mielke MM, et al. Higher risk of progression to dementia in mild cognitive impairment cases who revert to normal. Neurology 2014;82:317-25.

16. Foureur N, Mignot S, Senet P, et al. Correlation between the presence of type-2 anti-pemphigoid antibodies and dementia in elderly subjects with no clinical signs of pemphigoid. Ann Dermatol Venereol 2006;133:439-43.

17. Bastuji-Garin S, Joly P, Lemordant P, et al. Risk factors for bullous pemphigoid in the elderly: a prospective casecontrol study. J Invest Dermatol 2011;131:637-43.

18. Fichel F, Barbe C, Joly P, et al. Clinical and immunologic factors associated with bullous pemphigoid relapse during the first year of treatment: a multicenter, prospective study. JAMA Dermatol 2014;150:25-33.

19. Venning VA, Taghipour K, Mohd Mustapa MF, et al. British Association of Dermatologists' guidelines for the management of bullous pemphigoid 2012. Br J Dermatol 2012;167:1200-14.

20. Boessen R, Groenwold RH, Knol MJ, et al. Comparing HAMD(17) and HAMD subscales on their ability to differentiate active treatment from placebo in randomized controlled trials. J Affect Disord 2013;145:363-9.

21. Wang ZY, Zhang YM, Qu FY, et al. The application of Chinese Mini-mental state examination. Shanghai Archives of Psychiatry 1989;7:108-11.

22. Wen HB, Zhang ZX, Niu FS, et al. The application of Montreal cognitive assessment in urban Chinese residents of Beijing. Zhonghua Nei Ke Za Zhi 2008;47:36-9.

23. Ciesielska N, Sokołowski R, Mazur E, et al. Is the Montreal Cognitive Assessment (MoCA) test better suited than the Mini-Mental State Examination (MMSE) in mild cognitive impairment (MCI) detection among people aged

Cite this article as: Zhao W, Wang $\mathrm{Y}$, Mao X, Payne AS, Feng S, Li W, Wang Y, Wang D, Jin H, Yuan J, Li L. Detection of underlying dementia in bullous pemphigoid patients using cognitive evaluation tests: a multicenter case-control study. Ann Transl Med 2020;8(21):1397. doi: 10.21037/atm-20-1319 over 60? Meta-analysis. Psychiatr Pol 2016;50:1039-52.

24. Mitchell AJ. A meta-analysis of the accuracy of the mini-mental state examination in the detection of dementia and mild cognitive impairment. J Psychiatr Res 2009;43:411-31.

25. Janelidze M, Mikeladze N, Bochorishvili N, et al. Validity of the Georgian Montreal Cognitive Assessment for the Screening of Mild Cognitive Impairment and Dementia. Am J Alzheimers Dis Other Demen 2017;32:36-40.

26. Sun Q, Wang ZK, Lv XH. Cognitive impairment in patients with chronic cerebral circulation insufficiency assessed by MMSE and MoCA scale. J Apoplexy and Nervous Diseases 2016;33:143-5.

27. Zhai YL, Chao QL, Shen MZ, et al. Reliability and validity of Montreal cognitive assessment recension of Chinese version in elderly patients with mild cognitive impairment. Chin J Neuromed 2013;12:179-82.

28. Sun YC, Qin B. The application of Montreal cognitive assessment in amnestic mild cognitive impairment. Chin J Neuroimmunol 2011;18:91-3, 97.

29. Hugo J, Ganguli M. Dementia and Cognitive Impairment: Epidemiology, Diagnosis, and Treatment. Clin Geriatr Med 2014;30:421-42.

30. Jia JP, Wang YH, Zhang ZX, et al. Chinese guidelines for diagnosis and management of cognitive impairment and dementia(III): dementia subtypes and their criteria. Zhonghua Yi Xue Za Zhi 2011;91:735-41.

31. Messingham KN, Miller AD, Narayanan NS, et al. Demographics and Autoantibody Profiles of Pemphigoid Patients with Underlying Neurologic Diseases. J Invest Dermatol 2019;139:1860-1866.e1.

32. Mariotti F, Grosso F, Terracina M, et al. Development of a novel ELISA system for detection of anti-BP180 IgG and characterization of autoantibody profile in bullous pemphigoid patients. Br J Dermatol 2004;151:1004-10.

33. Expert group of experts on prevention and treatment of cognitive impairment in China. Expert consensus on prevention and treatment of cognitive impairment in China. Chin J Intern Med 2006;45:171-3. 